\title{
CONTROLE DO GORGULHO-DA-CANA SPHENOPHORUS LEVIS COM FUNGOS ENTOMOPATOGÊNICOS EM DIFERENTES DOSES E FORMULAÇÕES
}

VINHA, Fernando Belezini ${ }^{1}$ RODRIGUES, Luis Rodolfo ${ }^{2}$ PINTO, Alexandre De Sene ${ }^{3}$

\begin{abstract}
RESUMO: Os métodos de controle do gorgulho-da-cana-de-açúcar, Sphenophorus levis, existentes são pouco eficazes e pouco se conhece sobre o controle biológico dessa praga. Os fungos entomopatogênicos Metarhizium anisopliae e Beauveria bassiana infectam o gorgulho-da-cana, mas nem sempre são eficazes no campo, carecendo de informações de doses e formulações dos produtos. Portanto, esse trabalho teve como objetivo avaliar a eficácia dos fungos $M$. anisopliae e B. bassiana, em diferentes doses e formulações, no controle do $S$. levis, na cultura da canade-açúcar. O ensaio foi instalado em 28/05/2014 e conduzido na Usina Moreno, em Luís Antônio, SP, na variedade comercial RB 855035 (cana-soca). Em um delineamento em blocos casualizados, 8 tratamentos foram repetidos 3 vezes, em parcelas de $225 \mathrm{~m}^{2}$, sendo eles o fungo M. anisopliae GR, equivalente a 10 e $15 \mathrm{Kg} \mathrm{ha}^{-1}$ (conídios + arroz) e EC (diluído em óleo), nas doses equivalentes a 1,0 e 1,5 $\mathrm{L} \mathrm{ha}^{-1}$ e o fungo B. bassiana EC (diluído em óleo), equivalente a $0,5,1,0$ e 1,5 ha ${ }^{-1}$, além de uma testemunha (sem controle). Os fungos foram aplicados sobre o solo das soqueiras colhidas, manualmente (GR) ou com um pulverizador costal manual (EC). A área apresentava uma infestação média de 17,6 \pm 7,5\% de colmos atacados, 0,5 \pm 0,3 larvas e 0,1 \pm 0,1 adultos do gorgulho-da-cana por metro. Os dois fungos, nas doses e formulações utilizadas, e aplicados em uma época com baixa precipitação pluviométrica, foram pouco eficazes no controle de larvas e adultos do gorgulho-da-cana.
\end{abstract}

Palavras-chave: Praga agrícola. Controle microbiano. Curculionidae. Metarhizium anisopliae. Beauveria bassiana.

\section{CONTROL OF THE SUGARCANE WEEVIL SPHENOPHORUS LEVIS WITH ENTOMOPATHOGENIC FUNGI IN DIFFERENT DOSES AND FORMULATIONS.}

\begin{abstract}
SUMMARY: The existing methods of control of the sugarcane weevil, Sphenophorus levis, are weak and little is known about the biological control of this pest. The entomopathogenic fungi Metarhizium anisopliae and Beauveria bassiana infect the sugarcane weevil, but are not always effective in the field, lacking information about doses and formulations of these products. Therefore, this study aimed to evaluate the efficacy of the fungi M. anisopliae and B. bassiana at different doses and formulations in the control of S. levis, in the sugarcane crop. The trial was conducted on 2014 May, $28^{\text {th }}$ in the Moreno Mills, Luís Antônio, SP, Brazil, using the commercial variety RB 855035 (ratoon). In a randomized block design, eight treatments were replicated 3 times in plots of $225 \mathrm{~m}^{2}$. The treatments were the fungus $M$. anisopliae GR, equivalent to 10 is $15 \mathrm{~kg} \mathrm{ha}^{-1}$ (rice + conidia) and EC formulation (diluted in oil) at doses equivalent to 1.0 and $1.5 \mathrm{~L} \mathrm{~h}^{-1}$ and the fungus $B$. bassiana EC (diluted in oil), equivalent to $0.5,1.0$ and $1.5 \mathrm{ha}^{-1}$, and a control. The fungi were applied manually (GR) or with a manual sprayer (EC) on the soil of harvested clump. The area had an average infestation of $17.6 \pm 7.5 \%$ of stems attacked, $0.5 \pm 0.3$ larvae and $0.1 \pm 0.1$ adults of the weevil per meter. Both fungi, at doses and formulations used and applied in a season with low rainfall, were ineffective in controlling larvae and adults of the sugarcane weevil.
\end{abstract}

Keywords: Crop pest. Microbial control. Curculionidae. Metarhizium anisopliae. Beauveria bassiana.

\footnotetext{
${ }^{1}$ Graduado em Agronomia no CUML, Mestre em Entomologia pela ESALQ-USP.- Pesquisador na Biocontrol

${ }^{2}$ Centro Universitario Moura Lacerda - Graduação em Agronomia no CUML - Pesquisador na Occasio

${ }^{3}$ Centro Universitario Moura Lacerda - Graduado em Agronomia e Mestrado em Entomologia pela UNESP-FCAV, Doutorado em Entomologia pela ESALQ-USP - consultor agricola e professor no Centro Universitario Moura Lacerda
} 


\section{INTRODUÇÃO}

O Brasil é o maior produtor de cana-de-açúcar do mundo, sendo que na safra 2017 atingiu 8,73 milhões de hectares plantados e 657,18 milhões de toneladas produzidas (produtividade média de 72,6 t $\mathrm{ha}^{-1}$ ) (UDOP, 2017). A cana-de-açúcar é um agroecossistema que abriga numerosas espécies de insetos, sendo que algumas delas, dependendo da época do ano e da região, podem ocasionar sérios prejuízos econômicos (PARRA et al.,2010).

O gorgulho-da-cana, Sphenoporus levis Vaurie, 1978 (Coleoptera: Curculionidae), ataca as lavouras de cana-de-açúcar e é considerado limitante à produção da cultura em algumas regiões do Estado de São Paulo (ALMEIDA, 2005). Segundo Degaspari et al. (1987), as principais perdas são na produção, podendo chegar a 30 toneladas por hectare por ano. Na cana-planta com 5 a 7 meses de idade, cerca de 50 a $60 \%$ dos perfilhos podem ser mortos por causa da praga (PRECETI; ARRIGONI, 1990). Degaspari et al. (1987) afirmam que as larvas é quem causam danos, atacando os internódios basais do colmo, construindo galerias no seu interior, levando à morte das touceiras.

De acordo com Precetti e Arrigoni (1990) e Almeida (2005), até aquele momento não existiam inseticidas registrados para o controle da praga. Hoje existem alguns (AGROFIT, 2019), mas a eficácia ainda não é a mais adequada. A busca de agrotóxicos mais seletivos para utilização em lavouras é necessária para que eles não eliminem ou diminuam a ação dos inimigos naturais ou até promovam uma interação sinérgica quando utilizados em associação com os micro-organismos entomopatogênicos, contribuindo, assim, para um maior controle de determinada praga (CAVALCANTI et al., 2002).

O fungo Metarhizium anisopliae (Metschnikoff) Sorokin (Hypocreales: Clavicipitaceae) é amplamente distribuído na natureza e é encontrado facilmente nos solos, onde sobrevive por longos períodos. Este fungo pode colonizar mais de 300 espécies de insetos de várias ordens, sendo que nelas estão incluídas pragas de grande importância (ALVES, 1998). Alves et al. (2008) destacaram o uso desse fungo e de Beauveria bassiana (Balsamo) Vuillemin (Hypocreales: Cordycipitaceae), em iscas, para o controle de S. levis e Metamasius hemipterus (L., 1765) (Coleoptera: Curculionidae) em cana-de-açúcar, com até 92\% de eficiência (BADILLA; ALVES, 1991). Entretanto, utilizando-se iscas de colmos, na base de 200 por hectare, é uma tática inviável, necessitando ser aprimorada (ALVES et al., 2008).

Os fungos $M$. anisopliae e B. bassiana foram eficazes no controle de $S$. levis, em campo, quando aplicados na forma líquida ou granulada. Entretanto, M. anisopliae apresentou um poder residual mais longo do que o fungo B. bassiana, garantindo um melhor controle do gorgulho (DELFANTI, 2012; PAGLIARANI, 2012; SALVADOR NETO, 2012).

Salvador Neto (2012) concluiu que a melhor dose para o controle do gorgulho-da-cana com $M$. anisopliae GR foi de $4 \mathrm{Kg} \mathrm{ha}^{-1}$ e para B. bassiana de $6 \mathrm{Kg} \mathrm{ha}^{-1}$.

Este trabalho teve como objetivo avaliar a eficácia dos fungos $M$. anisopliae e B. bassiana no controle do S. levis, em diferentes doses e formulações, na cultura da cana-de-açúcar.

\section{MATERIAL E MÉTODO}

O ensaio foi instalado em 28 de maio de 2014, na Usina Moreno, em Luís Antônio, SP, em canavial comercial da variedade RB 855035 (cana-soca), com três cortes realizados. Os fungos entomopatogênicos $M$. anisopliae e $B$. bassiana utilizados no ensaio foram fornecidos pela Biotech controle biológico Ltda., Ribeirão Preto, SP. Os fungos foram produzidos sobre arroz.

O delineamento experimental foi realizado em blocos casualizados, onde cada um dos 8 


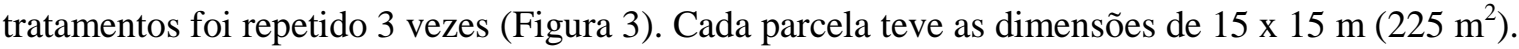

Os tratamentos foram: (1) fungo entomopatogênico $M$. anisopliae GR, na dose de $10 \mathrm{Kg} \mathrm{ha}^{-1}$ (conídios + arroz) $\left(6 \times 10^{13}\right.$ conídios viáveis ha $\left.{ }^{-1}\right)$; (2) M. anisopliae GR, $15 \mathrm{Kg} \mathrm{ha}^{-1}\left(9 \times 10^{13}\right.$ conídios viáveis ha $\left.{ }^{-1}\right)$; (3) M. anisopliae EC em óleo, $1,0 \mathrm{~L} \mathrm{ha}^{-1}\left(9 \times 10^{13}\right.$ conídios viáveis ha $\left.{ }^{-1}\right)$; (4) M. anisopliae EC em óleo, 1,5 L ha ${ }^{-1}\left(1,3 \times 10^{14}\right.$ conídios viáveis ha $\left.{ }^{-1}\right)$; (5) fungo entomopatogênico B. bassiana EC em óleo, $0,5 \mathrm{~L} \mathrm{ha}^{-1}\left(0,4 \times 10^{13}\right.$ conídios viáveis ha $\left.{ }^{-1}\right)$; (6) B. bassiana EC em óleo, 1,0 L ha ${ }^{-1}\left(0,8 \times 10^{13}\right.$ conídios viáveis ha $\left.{ }^{-1}\right)$; (7) B. bassiana $\mathrm{EC}$ em óleo, $1,5 \mathrm{~L} \mathrm{ha}^{-1}\left(1,2 \times 10^{14}\right.$ conídios viáveis ha $\left.{ }^{-1}\right)$; (8) testemunha (sem controle).

Figura 1. Esquema da distribuição dos blocos na área experimental. Luís Antônio, SP, 2014.

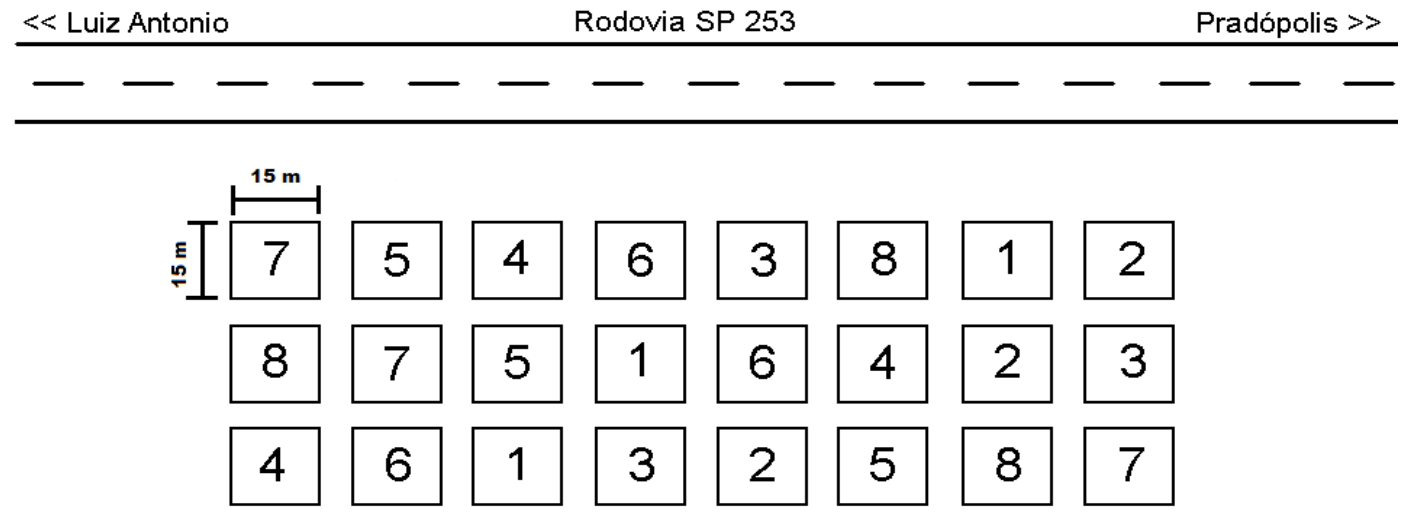

Os fungos foram aplicados no final da tarde por quatro pessoas aplicando doses diferentes ao mesmo tempo, para maior rendimento no trabalho. A aplicação da formulação GR foi feita manualmente, distribuindo os grãos com conídios sobre as touceiras, e da formulação EC, em pulverizadores costais manuais, utilizando o equivalente a $200 \mathrm{~L} \mathrm{ha}^{-1}$ de água.

Foram realizadas avaliações prévia e 60, 90 e 120 dias após a aplicação dos tratamentos. Em dois pontos escolhidos ao acaso dentro de cada parcela foram vistoriados todos os internódios basais e pedaços de colmos ("tocos") em um metro linear. Foi anotado o número de internódios basais ou "tocos" de colmos e o número daqueles com sintoma de ataque do gorgulho-da-cana e o número de larvas, pupas ou adultos de $S$. levis.

Calculou-se a porcentagem média de colmos danificados para cada tratamento e fez-se a correção (\% RC) desse valor pela fórmula de Henderson e Tilton (1955), que leva em consideração os danos para cada tratamento antes e após a aplicação dos mesmos. A fórmula utilizada foi:

$$
\% R C=100-\left(\frac{\left(n^{\circ} \text { na testemumha na prévia } \mathrm{x} \mathrm{n}^{\circ} \text { no tratamento na data }\right)}{\left(\mathrm{n}^{\circ} \text { na testemumha na data } \mathrm{x} \mathrm{n}^{\circ} \text { no tratamento na prévia }\right)} \times 100\right)
$$

Os dados obtidos foram transformados em médias e, posteriormente, estes foram submetidos à análise de comparação de médias pelo teste de Tukey ao nível de 5\% de significância.

\section{RESULTADO E DISCUSSÃO}

A área experimental escolhida para aplicar os tratamentos tinha, em 28/05/2014 (avaliação prévia, infestação média de 17,6 $\pm 7,5 \%$ de colmos atacados, mas apresentava apenas $0,5 \pm 0,3$ larvas e 0,1 $\pm 0,1$ 
adultos do gorgulho-da-cana, S. levis, em média, nos colmos e solo de 1 metro linear de cana-de-açúcar.

Quanto à porcentagem média de danos nos colmos causados por larvas de S. levis, houve diferenças significativas entre os tratamentos apenas aos 60 dias após a aplicação dos fungos. Nessa data, o tratamento que utilizou M. anisopliae EC na dose 1,5 $\mathrm{L} \mathrm{ha}^{-1}$ apresentou o maior valor e diferindo apenas daquele que utilizou o mesmo fungo EC em dose menor (Figura 2).

Calculou-se a porcentagem média de redução dos danos nos colmos, mas apenas 60 dias após a aplicação em que houve alguma eficácia. Pôde-se verificar que todos os fungos tiveram eficácia inferior a $50 \%$, sendo que B. bassiana EC 1,0 L ha ${ }^{-1}$ apresentou o maior valor (Figura 3).

Figura 2.Porcentagem média de colmos atacados por Sphenophorus levis após a aplicação dos fungos Metarhizium anisopliae e Beauveria bassiana, em diferentes doses ou formulações, em canavial após colheita. Luís Antônio, SP, 2014. Colunas seguidas pela mesma letra não diferem entre si pelo teste de Tukey $(\mathrm{p} \leq 0,05)$.

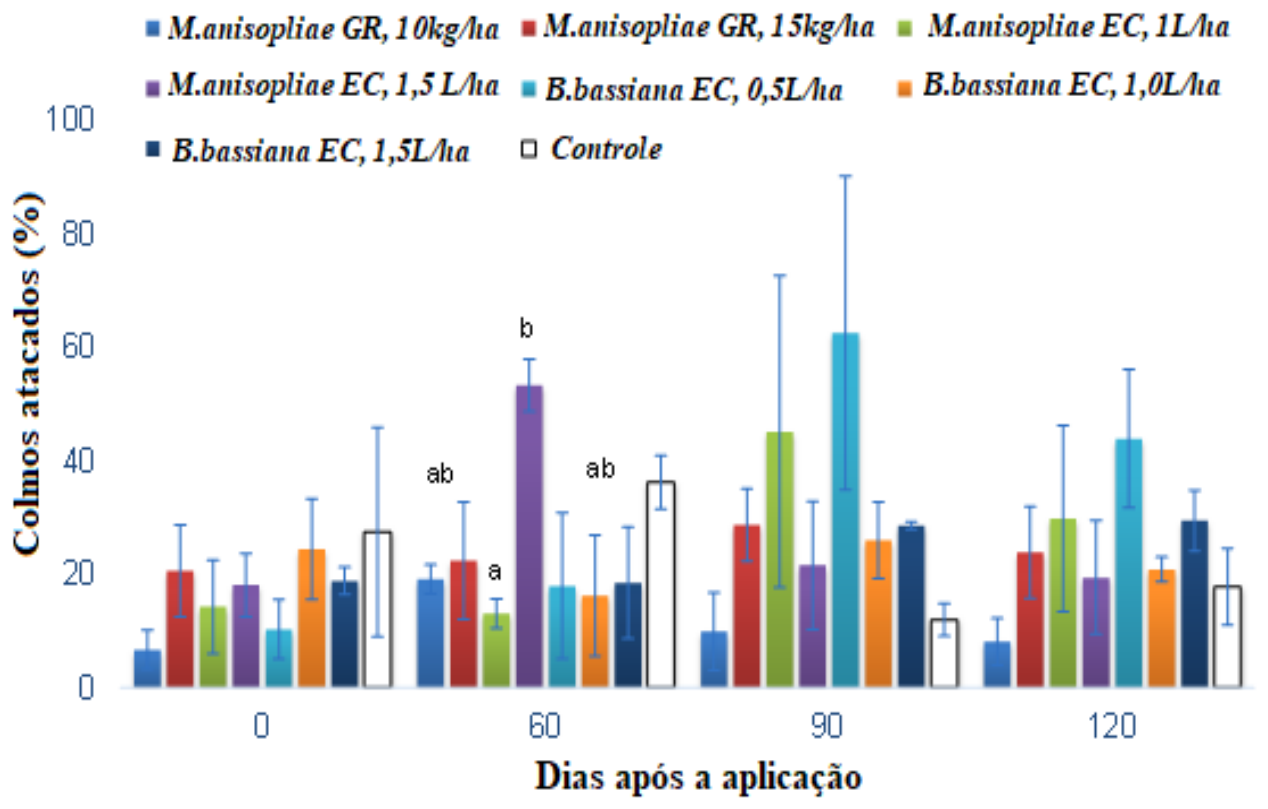

Figura 3.Porcentagem média de redução corrigida dos danos nos colmos atacados por larvas de Sphenophorus levis após a aplicação dos fungos Metarhizium anisopliae e Beauveria bassiana, em diferentes doses e formulações, em canavial após colheita. Luís Antônio, SP, 2014.

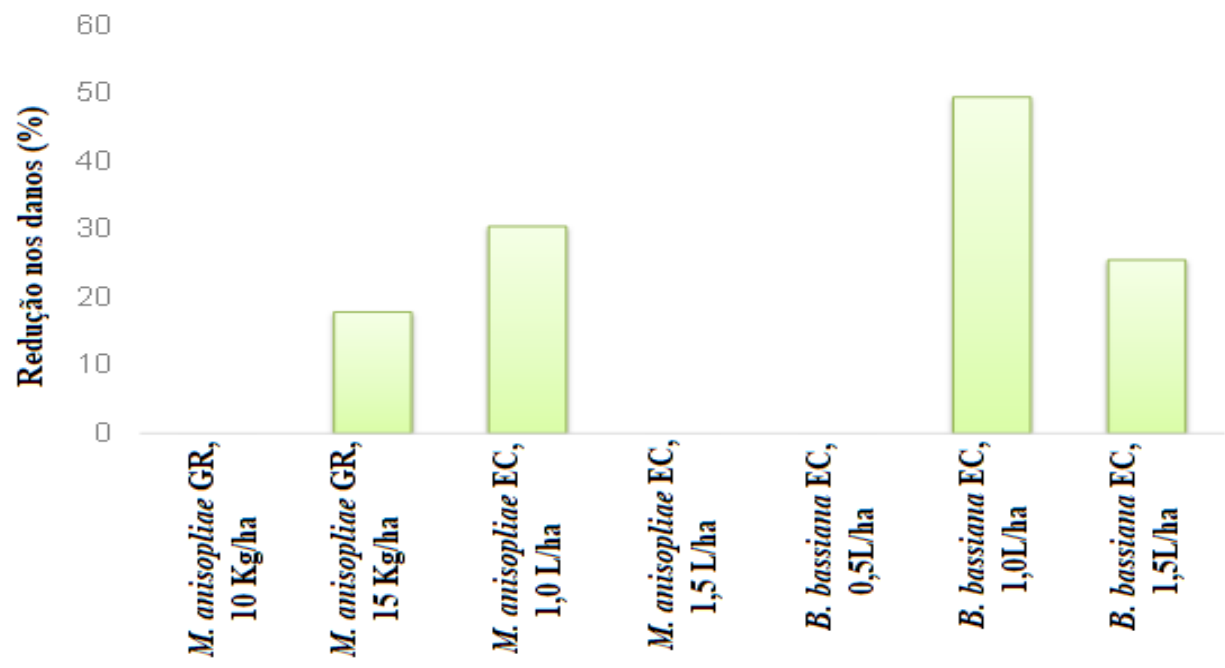


Os resultados foram diferentes dos obtidos anteriormente por outros autores, como Delfanti (2012), Pagliarani (2012) e Salvador Neto (2012). Também foram contrários à afirmação de Macedo, Garcia e Botelho (2006), em que o fungo M. anisopliae pode ser usado no controle do gorgulho-da-cana, e da confirmação de Badilla e Alves (1991), que obtiveram 92\% de controle de adultos de S. levis com o uso de toletes impregnados com B. bassiana.

Quanto ao número médio de larvas de S. levis em colmos e solo em um metro linear da linha de plantio, houve diferenças significativas entre os tratamentos apenas após 60 dias da aplicação dos fungos. Nessa data, nenhum dos tratamentos diferiu significativamente da testemunha, mas o tratamento $M$. anisopliae EC 1,5 $\mathrm{L} \mathrm{ha}^{-1}$ mostrou o maior valor, diferindo dos tratamentos igual em dose menor, $M$. anisopliae GR dose menor e dos tratamentos B. bassiana EC 0,5 e 1,0 $\mathrm{L} \mathrm{ha}^{-1}$ (Figura 4).

Figura 4. Número médio de larvas de Sphenophorus levis por colmos ou solo por metro linear após a aplicação dos fungos Metarhizium anisopliae e Beauveria bassiana, em diferentes doses ou formulações, em canavial após colheita. Luís Antônio, SP, 2014. Colunas seguidas pela mesma letra não diferem entre si pelo teste de Tukey $(\mathrm{p} \leq 0,05)$.

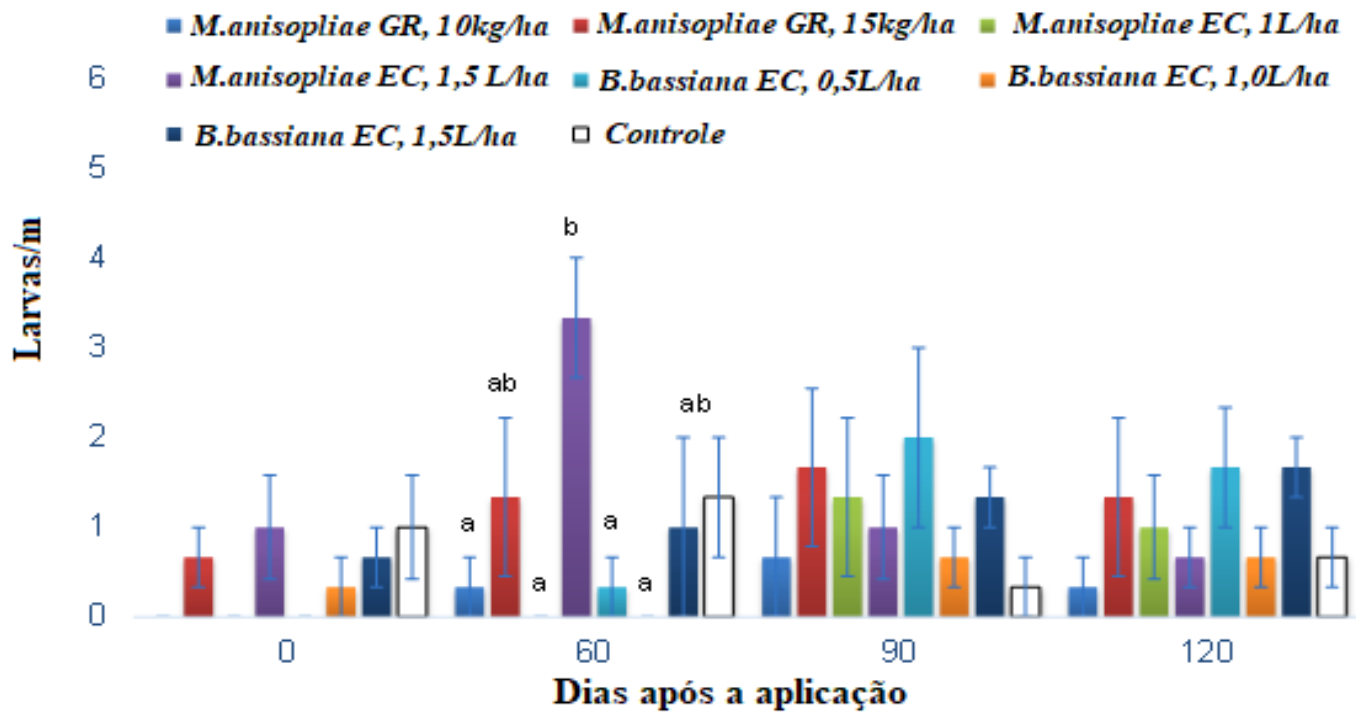

Entretanto, os fungos foram aplicados em uma época não muito favorável para a atuação desses micro-organismos, pois ocorreu baixa precipitação pluviométrica e as temperaturas estavam em queda.

Pedrão (2012), estudando o efeito do clima na eficácia dos fungos M. anisopliae e B. bassiana no controle de lagartas de Spodoptera frugiperda em milho concluiu que o fungo $M$. anisopliae é mais favorecido pelas precipitações pluviométricas que ocorrem no momento da aplicação e que B. bassiana foi mais eficaz quando aplicado em períodos sem chuva, apesar de uma ação mais lenta.

Rodas (2006) também verificou baixa eficácia de fungos entomopatogênicos no controle de Praelongaorthezia praelonga (=Orthezia praelonga) (Douglas) (Hemiptera: Ortheziidae) quando aplicados em período seco, mas também verificou a melhor eficácia (ao redor de $50 \%$ ) do fungo $B$. bassiana nessa condição.

Novos ensaios em locais distintos e em épocas diferentes deverão ser conduzidos para tentar mostrar a eficácia de $M$. anisopliae e B. bassiana no controle de $S$. levis. Esses ensaios também permitirão compreender melhor o efeito do clima na eficiência de controle do fungo, também os diferentes modos de aplicação deverão ser levados em consideração em novas pesquisas. 


\section{CONCLUSÃO}

Baseado nas condições em que o ensaio foi conduzido, ou seja, presença de danos expressivos da praga, clima e localidade, pode-se concluir que:

- Os fungos entomopatogênicos Metarhizium anisopliae e Beauveria bassiana aplicados na formulação granulada GR (arroz + conídios) ou líquida EC (em óleo) são pouco eficazes no controle do gorgulho-da-cana, Sphenophorus levis, em campo;

- O fungo B. bassiana EC 1,0 $\mathrm{L} \mathrm{ha}^{-1}$ é o que garante o melhor controle do gorgulho, porém com baixo período residual e eficácia.

\section{REFERÊNCIAS}

AGROFIT. Disponível em: <http://agrofit.agricultura.gov.br/agrofit_cons/principal_agrofit_cons> Acesso em: 15/01/2019.

ALMEIDA, L.C. Bicudo da cana-de-açúcar: informação técnica. Piracicaba: Centro de Tecnologia Canavieira, 2005. (Boletim Técnico)

ALVES, S.B. Fungos entomopatogênicos. In: ALVES, S.B. (Ed.). Controle microbiano de insetos, 2.ed. Piracicaba: Fealq, 1998. p.289-381.

ALVES, S.B.et al. Fungos entomopatogênicos usados no controle de pragas na América Latina. In: ALVES, S.B.; LOPES, R.B (Eds.). Controle microbiano de pragas na América Latina: avanços e desafios. Piracicaba: Fealq, 2008. p.69-110.

BADILLA, F.F.; ALVES, S.B. Controle do gorgulho da cana-de-açúcar Sphenophorus levis Vaurie, 1978 (Coleoptera: Curculionidae) com Beauveria spp. em condições de laboratório e campo. Anais da Sociedade Entomológica do Brasil, v.20, n.1, p.251-262, 1991.

CAVALCANTI, R.Set al. Efeito dos produtos fitossanitários fenpropatrina, imidaclopride, iprodione e tiametoxam sobre o desenvolvimento do fungo Beauveria bassiana (Bals.) Vuill. Arquivos do Instituto Biológico, v.69, n.3, p.17-22, 2002.

DEGASPARI, N.et al. Biologia de Sphenophorus levis (Vaurie, 1978) (Coleoptera: Curculionidae) em dieta artificial e no campo. Pesquisa Agropecuária Brasileira, v.22, n.6, p.556-558, 1987.

DELFANTI, L.A. de A. Eficácia do fungo Metarhizium anisopliae no controle de Sphenophorus levis e efeito em outras pragas de solo em cana-de-açúcar. 2012. 30f. Monografia (Trabalho de Conclusão de Curso em Agronomia) - Centro Universitário Moura Lacerda, Ribeirão Preto.

HENDERSON, C.F.; TILTON, E.W. Tests with acaricides against the brown wheat mite. Journal of Economic Entomology, v.48, p.157-161, 1955.

MACEDO, L.P.M.; GARCIA, J.F.; BOTELHO, P.S.M. Outros besouros-praga da cana-de-açúcar. In: PINTO, A. de S. (Org.). Controle de pragas da cana-de-açúcar. Sertãozinho: Biocontrol, 2006. p.49-52.

PAGLIARANI, V.D. Formulação e dose do fungo Beauveria bassiana no controle de Sphenophorus levis e efeito em pragas de solo em cana-de-açúcar. 2012. 23f. Monografia (Trabalho de Conclusão de Curso em Agronomia) - Centro Universitário Moura Lacerda, Ribeirão Preto. 
PARRA, J.R.P.; BOTELHO, P.S.M.; PINTO, A. de S. Controle biológico de pragas como um componente-chave para a produção sustentável da cana-de-açúcar. In: CORTEZ, L.A.B. (Org.). Bioetanol de cana-de-açúcar: P\&D para produtividade e sustentabilidade. São Paulo: Blucher, 2010. p.441-450.

PEDRÃO, V. O clima influenciando a eficiência de fungos entomopatogênicos no controle de Spodoptera frugiperda (Lepidoptera: Noctuidae) em milho. 2012. 32f. Monografia (Trabalho de Conclusão de Curso em Agronomia) - Centro Universitário Moura Lacerda, Ribeirão Preto.

PRECETTI, A.A.C.M.; ARRIGONI, E.B. Aspectos bioecológicos e controle do besouro Sphenophorus levis Vaurie, 1978 (Coleoptera, Curculionidae) em cana-de-açúcar. São Paulo: Copersucar, 1990. 15p. (Boletim Técnico Copersucar - Edição Especial)

RODAS, T.H.Z. Utilização de fungos entomopatogênicos no controle de Orthezia praelonga Douglas, 1891 (Hemiptera: Ortheziidae) em pomar de citros orgânico. 2006. 23f. Monografia (Trabalho de Conclusão de Curso em Agronomia) - Centro Universitário Moura Lacerda, Ribeirão Preto.

SALVADOR NETO, N. Doses dos fungos Metarhizium anisopliae e Beauveria bassiana GR no controle de Sphenophorus levis e corós em cana-de-açúcar. 2012. 25f. Monografia (Trabalho de Conclusão de Curso em Agronomia) - Centro Universitário Moura Lacerda, Ribeirão Preto.

UDOP - UNIÃO DOS PRODUTORES DE BIOENERGIA. Evolução da produtividade cana-de-açúcar safras 2005 a 2017. Disponível em:

$<\mathrm{http} / /$ wwww.udop.com.br/download/estatistica/area_cultivada/2017_nov_regiao_brasileira_area_plantada. pdf> Acesso em: 14/11/2017. 